\title{
Does System 1 Process both Local and Nonlocal Information in Intuitive Judgment and Decision Making? Available Evidence and a Research Agenda Proposal
}

\author{
Patrizio Tressoldi \\ Dipartimento di Psicologia Generale, Università di Padova, Padova, Italy \\ Email: patrizio.tressoldi@unipd.it
}

Received July 31 $1^{\text {st }}, 2013$; revised September $1^{\text {st }}, 2013$; accepted October $2^{\text {nd }}, 2013$

\begin{abstract}
Copyright (C) 2013 Patrizio Tressoldi. This is an open access article distributed under the Creative Commons Attribution License, which permits unrestricted use, distribution, and reproduction in any medium, provided the original work is properly cited.
\end{abstract}

\begin{abstract}
This paper argues that System 1, (the mental processing system mainly involved in the processing of unconscious information) in contrast to System 2, (mainly involved in the processing of conscious information), processes not only local information conveyed by sensory organs, but also nonlocal ones, that is, those beyond the detection range of sensory organs. The striking similarities observed between the characteristics of local and nonlocal information processing by System 1, offer the possibility of using most of the experimental protocols used to investigate local information for the nonlocal information. Available evidence is presented and a research agenda is outlined that could raise fascinating questions and answer about the functioning of the human mind.
\end{abstract}

Keywords: System 1; System 2; Unconscious Information Processing; Nonlocal Information

\section{Introduction}

\section{Dual-Process Model of Information Processing: System 1 and System 2}

Although some authors have traced the dual-process theory of information processing to William James, a consensus has been achieved on the theoretical and empirical definition of two distinct ways of perceiving and thinking that characterize the functioning of human mental activity, System 1 and System 2 as defined by Epstein (1994, 2008); Stanovich (1999); Kahneman, (2003, 2011); Kahneman \& Frederick (2002); Evans \& Stanovich (2013).

These information processing systems can be summarised as the extremes of a continuum with System 1 as a fast, automatic, emotional, stereotypic, large capacity, sub- and unconscious way of processing at one end, and System 2 as a slow, effortful, capacity limited, controlled and conscious way of processing information at the other.

There is some evidence that System 1's unconscious cognitive activity influences consumer preferences (Friese, Wänke, \& Plessner, 2006), interpersonal processes (Fitzsimons \& Bargh, 2003), emotional reactions (Tamietto et al., 2009) and the pursuit of specific goals (Custers \& Aarts, 2010).

A useful and clearly expressed comparison of the main characteristics of System 1 and System 2 within four different clusters, is offered by Evans (2008) ${ }^{1}$, see Table 1.

At present, the theoretical and empirical investigation is largely devoted to the exploration of the conditions that trigger the activation of one system or the other, (e.g. Greifeneder, Bless, \& Pham, 2011; Bolte \& Goschke, 2005; Evans \& Stanovich,

${ }^{1}$ A similar comparison has been done by Epstein (2008).
2013), defining which problems can be solved more efficiently by using one system or the other, (e.g. Usher et al., 2011), and determining if and how these two systems can interact to exploit their characteristics for increasing information processing efficiency (e.g. Evans, 2008; Kruglanski \& Gigerenzer, 2011).

What information do these two systems use? The general consensus is that all the information is received only by the sensory organs.

As strange as it may seem, there is a research line more than 80 years old, which investigates whether the human mind can process information received by bypassing the sensory organs (see Tressoldi, 2011, for a review) although it has only recently been theoretically and empirically examined within a dual-system framework. Even if it pursued under different names and theoretical approaches, we can define this as the study of System 1 nonlocal information processing. In the following sections we define the characteristics of this information processing system. We then outline the empirical findings obtained so far and conclude with a proposal for a research agenda to pursue investigation of the interactions in the processing systems of both local and nonlocal information.

\section{Could System 1 Process Nonlocal Information?}

Suppose our brain and consequently our mind could receive information related to objects, people, emotions, natural envi-

\footnotetext{
${ }^{2}$ The meaning of "nonlocal" is used here only as presented in the text and not as physical or biological quantum-like properties. An interpretation of mental nonlocal functions as possible physical or biological quantum-like properties is presented in Tressoldi \& Khrennikov (2012).
} 


\section{P. TRESSOLDI}

Table 1.

Clusters of attributes associated with dual systems of thinking following Evans (2008).

\begin{tabular}{cc}
\hline System 1 & System 2 \\
\hline Cluster 1-Consciousness & \\
Unconscious (preconscious) & Conscious \\
Implicit & Explicit \\
Automatic & Controlled \\
Low effort & High effort \\
Rapid & Slow \\
High capacity & Low capacity \\
Default process & Inhibitory \\
Holistic, perceptual & Analytic, reflective \\
Cvolutionarily old & \\
Evolutionary rationality & Evolutionarily recent \\
Shared with animals & Individual rationality \\
Nonverbal & Uniquely human \\
Modular cognition & Linked to language
\end{tabular}

Cluster 3-Functional characteristics

$\begin{array}{cc}\text { Associative } & \text { Rule based } \\ \text { Domain specific } & \text { Domain general } \\ \text { Contextualized } & \text { Abstract } \\ \text { Pragmatic } & \text { Logical } \\ \text { Parallel } & \text { Sequential } \\ \text { Stereotypical } & \text { Egalitarian }\end{array}$

Cluster 4-Individual differences

Universal Heritable

Independent of general intelligence Linked to general intelligence

Independent of working memory Limited by working memory capacity

ronment, etc., from beyond the constraints of our sensory organs by acting like an information detector. In simple terms, this would involve expressing nonlocal perception characteristics in different ways from the perception of information transmitted by our sensory organs which are limited by their biological detection range.

We will call this System 1 nonlocal information processing (S1_nip) postulating that its activity precedes System 1 local processing (S1_lip) and obviously System 2 activity.

The hypothesis of a mind or consciousness without space and perhaps time constraints is not new and we can trace its roots back to certain philosophical theories, such as idealism, panpsychism, neutral or mental monism and more recently nonphysical realism (Staune, 2013) and the dual-aspect monism
(Antmaspacher, 2012).

For example, Idealism is the family of views which assert that reality, or reality as we know it, is fundamentally mental, mentally constructed, or otherwise immaterial (e.g. Bolender, 2001).

Panpsychism states that mind is a fundamental feature of the world which exists throughout the universe. Unsurprisingly, each of the key terms, "mind", "fundamental" and "throughout the universe" is subject to a variety of interpretations by panpsychists, leading to a range of possible philosophical positions (Pihlström, 2008).

Mental Monism holds that all is mind and that the ultimate constituents of the world are individual momentary experiences which in themselves are neither mental nor physical, but of which, differently arranged, both minds and material things are composed. In other words, the concept of nature itself is a construct of mind that can only be known through hypotheses tested by reference to experience (Velmans, 2012).

Non-physical realism refers to the assumption that reality cannot be explained exclusively by observable causes in spacetime and consciousness and matter stem from a unique substance that would "ante-date the scission between the subject and the objet" (Staune, 2013) a theoretical approach similar to the dual-aspect monism (Antmaspacher, 2012) elaborated after a detailed reconstruction of the Pauli-Jung conjecture which yields a psychophysically neutral, unitary reality beyond the distinction of the mental and the material.

In psychology, the term and characteristics of nonlocal mind or consciousness were first proposed by Dossey (1989) and more recently by Clarke (1995) and Carpenter $(2004,2012)$ in his First Sight Model.

This paper is the first systematic proposal to introduce this model of processing within the dual-model framework.

Which nonlocal information can be received by S1 nip? In theory it should be all the information available in the universe regardless of distance and perhaps time constraints. But how can a living mind survive this massive flow of information? One possibility, supported by evidence (see Section 2), is that this information has a very low signal-to-noise ratio and in the normal condition of the mind functioning in a waking status of consciousness, System 2 and S1_lip functioning will almost completely mask nonlocal information signals. A good analogy is our daily immersion in the ocean of information transmitted by electromagnetic waves that we are unaware of until we decide to perceive them by tuning our detectors, radio, smartphones, etc. to amplify their signal strength to a level compatible with our sensory organs' detection characteristics.

If this assumption is correct, we should observe better detection of nonlocal information reducing or bypassing the activity of S1_lip and System 2 processing of local information. How is it possible to reduce or bypass S1_lip and System 2 activity?

One possibility is to reduce or eliminate completely the flow of information sent by the sensory organs and the information processing of S1_lip and System 2. For example, to reduce the flow of information sent by the sensory organs, participants in experiments have been immersed in a ganzfeld environment (see a description of a typical protocol in the Appendix). Another alternative to bypassing S1 lip and System 2 activity is to record the neuro- or psychophysiological signals correlated with nonlocal information (see description in the Appendix). With these two experimental protocols, the supposedly non-local information may be detected consciously because any other 


\section{P. TRESSOLDI}

mental activity of S1 lip and System 2 is maximally reduced in a ganzfeld environment, whereas with the second protocol it is possible to detect directly the purported nonlocal information at an unconscious level when it impinges on the central or peripheral nervous system.

\section{Evidence $^{3}$}

It is relevant at this point to ask what the differences are in S1_nip detection accuracy for nonlocal information with different degrees of S1_lip and Sensory 2 involvement. In Table 2, we list the best evidence available from meta-analyses in decreasing order of effect size (ES) values expressed as Cohen's $d$ standardised difference. Except for the "anticipatory physiological signals" where ES is the difference between the two stimulus categories, in all of the other experimental conditions, ES is the difference with respect to the expected chance.

From the data presented in Table 2, it is clear that the detection of information of S1_nip decreases almost linearly, passing from a condition where $\mathrm{S} 1$ lip and System 2 are bypassed, i.e. in dream status or when recording neuro- and psychophysiological signals, to a condition where S1_lip and System 2 activity is reduced, i.e. in a ganzfeld environment or in an altered state of consciousness, to conditions in which nonlocal information is almost completely lost, i.e. in normal status of consciousness when S1 lip and System 2 are both active, independently if free or forced-choice procedures are used. In summary, the more System 2 and S1_lip are activated, the more nonlocal information is destroyed (suppressed) in analogy with a situation where we try to understand what a friend is telling us in a disco.

If we compare the efficiency of S1_lip in some experimental conditions, we can see how much stronger is its detection accuracy with respect to $\mathrm{S} 1 \mathrm{nip}$ (see Table 3 ).

Similarly to what has been observed for S1_nip, we can see

Table 2.

Experimental evidence obtained from meta-analyses of S1 nip detection accuracy in decreasing order of ES values.

\begin{tabular}{cccc}
\hline $\begin{array}{c}\text { Experimental } \\
\text { condition }\end{array}$ & Source & N. Studies & $\begin{array}{c}\text { Weighted random } \\
\text { effect ES }(95 \% \mathrm{CI})\end{array}$ \\
\hline Dream & $\begin{array}{c}\text { Sherwood and } \\
\text { Roe (2003) }\end{array}$ & 22 & $.24(.20-.28)$ \\
$\begin{array}{c}\text { Anticipatory } \\
\text { physiological signals }\end{array}$ & $\begin{array}{c}\text { Mossbridge et al. } \\
(2012)\end{array}$ & 26 & $.21(.13-.29)$ \\
$\begin{array}{c}\text { Free response in } \\
\text { Ganzfeld }\end{array}$ & Tressoldi (2011) & 108 & $.13(.09-.17)$ \\
$\begin{array}{c}\text { Free-response in } \\
\text { ASC }\end{array}$ & Storm et al. (2010) & 16 & $.11(.03-.19)$ \\
$\begin{array}{c}\text { Implicit behavioral } \\
\text { anticipation effects } \\
\text { in NSC }\end{array}$ & $\begin{array}{c}\text { Tressoldi et al. } \\
\text { (submitted) }\end{array}$ & 82 & $.09(.06-.12)$ \\
$\begin{array}{c}\text { Forced-choice in } \\
\text { NSC }\end{array}$ & Tressoldi (2011) & 72 & $.01(.006-.015)$ \\
$\begin{array}{c}\text { Free-response in } \\
\text { NSC }\end{array}$ & Storm et al. (2010) & 14 & $-.03(-.06-.002)$ \\
\hline
\end{tabular}

Note: ${ }^{*} \mathrm{ASC}=$ altered states of consciousness, like hypnosis, deep meditation; ${ }^{* *} \mathrm{NSC}=$ normal state of consciousness.

${ }^{3}$ We are aware that the interpretation of this evidence (as support to the reality of nonlocal perception) is hotly debated, but there is not sufficient space in this paper to discuss all alternative explanations.
Table 3.

Some Experimental evidence obtained from meta-analyses of S1_lip detection accuracy in decreasing order of ES values.

\begin{tabular}{|c|c|c|c|}
\hline $\begin{array}{l}\text { Experimental } \\
\text { condition }\end{array}$ & Source & N. Studies & $\begin{array}{c}\text { Weighted random effect } \\
\text { ES }(95 \% \mathrm{CI})\end{array}$ \\
\hline $\begin{array}{c}\text { Semantic } \\
\text { categorization } \\
\text { priming }\end{array}$ & $\begin{array}{l}\text { Van den Bussche } \\
\text { et al. (2009) }\end{array}$ & 23 & $.80(.60-1.00)$ \\
\hline $\begin{array}{l}\text { Lexical and } \\
\text { naming priming }\end{array}$ & $\begin{array}{c}\text { Van den Bussche } \\
\text { et al. (2009) }\end{array}$ & 32 & $.47(.36-.58)$ \\
\hline $\begin{array}{l}\text { Problem solving } \\
\text { Incubation effect }\end{array}$ & $\begin{array}{l}\text { Sio \& Ormerod } \\
\text { (2009) }\end{array}$ & 117 & $.29(.20-.38)$ \\
\hline $\begin{array}{l}\text { Unconscious } \\
\text { thought theory }\end{array}$ & $\begin{array}{l}\text { Strick et al. } \\
\quad(2011)\end{array}$ & 92 & $.22(.14-.30)$ \\
\hline
\end{tabular}

that the S1 lip efficiency decreases when passing from conditions where System 2 is bypassed, i.e. priming, to conditions where System 2 is temporarily inhibited (Van den Bussche et al., 2009) or made busy during System 1 processing, i.e., problem-solving incubation and unconscious complex problem-solving tasks (i.e. Sio \& Ormerod, 2009; Strick et al., 2011).

The comparison of ESs presented in Tables 2 and 3, clearly shows the superior efficiency of S1 lip where the information is sent to the sensory organs in any case, with respect to S1_nip. The smallest efficiency values of S1 lip are close to the largest values of S1 nip, supporting the hypothesis that the nonlocal information signal-to-noise ratio is weak and hence its detection can easily be suppressed by S1_lip or System 2 processing activity, even if we cannot exclude the possibility that it still has a weak influence both of them.

\section{System 1 Local and Nonlocal Information Processing Comparison}

At this point it is possible to compare the characteristics of S1_nip and S1_lip using the same template used to compare S1_lip with System 2 (see Table 4).

The characteristics in bold are those assumed to be specific only to S1 nip. Those with a question mark, need to be tested.

A striking similarity emerges with regard to the characteristics of the two information processing systems. A few basic differences obviously exist in terms of their origin, receptive capacity, constraints imposed by sensory organs for S1_lip, unbounded for S1_nip and different interference vulnerability, partly consequent on the different signal-to-noise ratios that are higher for S1_lip.

\section{A Research Agenda}

The analysis of the characteristics of S1_lip and S1_nip, suggests that, apart from the basic difference in the source of information, they should function in a very similar way even if the effects on behaviour and System 2 are stronger for S1_lip and interference of System 2 is stronger for S1 nip than S1 lip.

It follows that most of the experimental protocols that are used to study S1_lip can be used to study S1_nip. For example, all the protocols used to study the priming effects with S1 lip can easily be adapted to study these effects, presenting both subliminal and completely masked priming information. For example, Figure 1 shows a classical example of semantic priming where the priming, the picture of a hammer in this case, is presented subliminally. If we present the picture of the hammer 
Table 4.

Similarities and differences between S1_nip and S1_lip.

\begin{tabular}{cc}
\hline S1_nip & S1_lip \\
\hline Unconscious (preconscious) & Unconscious (preconscious) \\
Implicit & Implicit \\
Automatic & Automatic \\
Low effort & Low effort \\
Rapid & Rapid \\
High capacity & High capacity \\
Default process & Default process \\
Holistic, perceptual & Holistic, perceptual
\end{tabular}

Cluster 2-Evolution

$\begin{array}{cc}\text { Evolutionarily innate } & \text { Evolutionarily old } \\ \text { Evolutionary rationality } & \text { Evolutionary rationality } \\ \text { Shared with animals } & \text { Shared with animals } \\ \text { Nonverbal } & \text { Nonverbal } \\ \text { Modular cognition } & \text { Modular cognition }\end{array}$

Cluster 3-Functional characteristics

$\begin{array}{cc}\text { Associative } & \text { Associative } \\ \text { Domain specific } & \text { Domain specific } \\ \text { Contextualized?? } & \text { Contextualized } \\ \text { Pragmatic } & \text { Pragmatic } \\ \text { Parallel } & \text { Parallel } \\ \text { Stereotypical?? } & \text { Stereotypical }\end{array}$

Cluster 4-Individual differences

Universal Universal

Independent of general intelligence Independent of general intelligence

Independent of working memory Independent of working memory

Cluster 5-Interaction with System 2

Affect System 2

Affect System 2

Affected by System 2

Affected by System 2

Cluster 6-Sensorial characteristics

$\begin{array}{cc}\begin{array}{c}\text { Unbounded by sensory organs } \\ \text { detection characteristics }\end{array} & \begin{array}{c}\text { Bounded by sensory organs } \\ \text { detection characteristics }\end{array} \\ \begin{array}{c}\text { Interference from information } \\ \text { out of sensory detection }\end{array} & \begin{array}{c}\text { No influence by information } \\ \text { out of sensory detection }\end{array} \\ \text { Very low signal/noise ratio } & \begin{array}{c}\text { Signal/noise ratio depends } \\ \text { from the duration and the } \\ \text { strength of sensory organs } \\ \text { stimulation }\end{array} \\ & \end{array}$

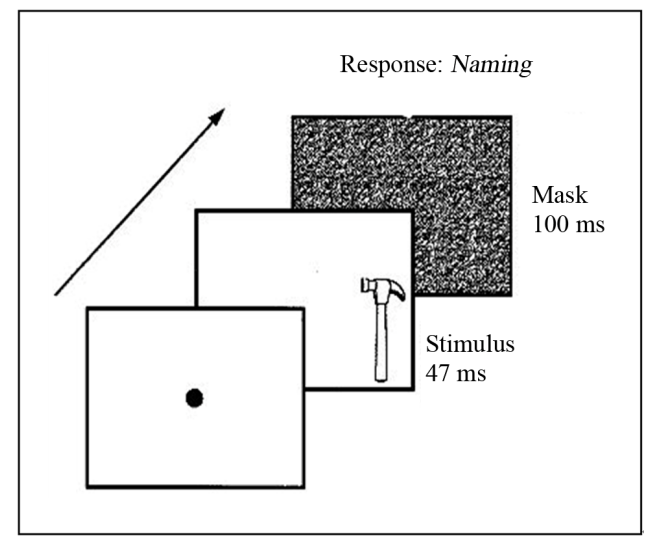

Figure 1.

Classical example of a procedure to investigate unconscious semantic priming.

completely covered, we can investigate whether naming can be primed by the nonlocal perception.

If our premises are valid, we should observe similar priming effects in both conditions even if they are weaker (i.e. lower effect sizes) associated with the presentation of nonlocal information.

One example on how to combine subliminal and nonlocal information, is offered by Carpenter, Simmonds-Moore and Moore (2012) that studied the Mere Exposure Effect, that is the tendency for persons to experience a greater liking or attraction for things as a function of having been exposed to them previously. These authors used both subliminal and nonlocal information by simply masking them completely during their presentation in a way that no sensory information is available.

Other functioning similarities are expected. For example it could be tested if semantic and perceptual object features presented out of the range of the detection of sensory organs, differentially modulates the sensitivity of unconscious processing pathways, producing similar effects of attentional induction such as those studied by Martens, Ansorge, \& Kiefer (2011).

The core of this research agenda should be the study of the relationships between these three information processing systems. Thus some of the main questions that need to be asked are:

- What are the adaptive advantages of having both an S1_lip and an S1_nip?

- Can an S1_lip and an S1_nip be used cooperatively to enhance their specific properties?

- What are the neuro- and psychophysiological correlates of an S1_lip and an S1_nip?

- How can we exploit the information processing of S1_nip, for example by detecting and amplifying the signal strength of its neuro- and psychophysiological correlates, to assist System 2 information processing?

- Are some so-called "anomalous" or "exceptional" perceptual and cognitive experiences a sign of enhanced S1_nip (e.g. Fach et al., 2013)?

- What is the role of implicit goals pursuit (Custers \& Aarts, 2010) and basic needs (see Epstein, 2008) in the facilitation or inhibition of S1_nip detection accuracy?

- Which individual differences facilitate the use of S1_lip and S1_nip information in avoiding the interference of System 2 (e.g. the role of Zen meditation, Strick et al., 2012)? 


\section{P. TRESSOLDI}

Many more questions are obviously possible, but their resolution depends on how many scientists are interested in exploring this frontier of the human mind.

\section{Acknowledgements}

English was independently revised by the Proof Reading Service and the Professional Editing Services

\section{REFERENCES}

Atmanspacher, H. (2012). Dual-aspect monism à la Pauli and Jung. Journal of Consciousness Studies, 19, 96-120.

Bolender, J. (2001). An argument for idealism. Journal of Consciousness Studies, 8, 37-61.

Bolte, A., \& Goschke, T. (2005). On the speed of intuition: Intuitive judgments of semantic coherence under different response deadlines. Memory \& Cognition, 33, 1248-1255.

http://dx.doi.org/10.3758/BF03193226

Carpenter, J. C. (2004). First sight: Part one, a model of psi and the mind. Journal of Parapsychology, 68, 217.

Carpenter, J. C. (2012). First sight. ESP and parapsychology in everyday life. New York: Rowman \& Littlefield Pub. Inc..

Carpenter, J. C., Simmonds-Moore, C., Moore, S., \& Carpenter, F. (2012). Extrasensory perception contributes to the formation of a preference: II. Confirmation of patterns found previously. Paper presented at the PA 55th Annual Convention, Durham, North Carolina August 9-12.

Clarke, C. J. S. (1995). The nonlocality of mind. Journal of Consciousness Studies, 2, 231-240.

Custers, R., \& Aarts, H. (2010). The unconscious will: How the pursuit of goals operates outside of conscious awareness. Science, 329, 4750. http://dx.doi.org/10.1126/science. 1188595

Dunne, B. J., \& Jahn, R. G. (2003). Information and uncertainty in remote perception research. Journal of Scientific Exploration, 17, $207-$ 241.

Dossey, L. (1989). Recovering the soul. New York, NY: Bantam.

Epstein, S. (1994). Integration of the cognitive and the psychodynamic unconscious. American Psychologist, 49, 709-724.

http://dx.doi.org/10.1037/0003-066X.49.8.709

Epstein, S. (2008). Intuition from the perspective of cognitive-experiential self-theory. In H. Plessner, C. Betsch, \& T. Betsch (Eds.), Intuition in judgment and decision making (pp. 23-37). New York: Lawrence Erlbaum Associates.

Evans, J. S. B. (2008). Dual-processing accounts of reasoning, judgment, and social cognition. Annual Review of Psychology, 59, $255-$ 278. http://dx.doi.org/10.1146/annurev.psych.59.103006.093629

Evans, J. S. B., \& Stanovich, K. E. (2013). Dual-process theories of higher cognition: Advancing the debate. Perspectives on Psychological Science, 8, 223-241. http://dx.doi.org/10.1177/1745691612460685

Fach, W., Atmanspacher, H., Landolt, K., Wyss, T., \& Rössler, W. (2013). A comparative study of exceptional experiences of clients seeking advice and of subjects in an ordinary population. Frontiers in Psychology, 4, 65. http://dx.doi.org/10.3389/fpsyg.2013.00065

Fitzsimons, G. M., \& Bargh, J. A. (2003). Thinking of you: Nonconscious pursuit of interpersonal goals associated with relationship partners. Journal of Personality and Social Psychology, 84, 148-163. http://dx.doi.org/10.1037/0022-3514.84.1.148

Friese, M., Wänke, M., \& Plessner, H. (2006). Implicit consumer preferences and their influence on product choice. Psychology \& Marketing, 23, 727-740. http://dx.doi.org/10.1002/mar.20126

Greifeneder, R., Bless, H., \& Pham, M. T. (2011). When do people rely on affective and cognitive feelings in judgment? A review. Personality and Social Psychology Review, 15, 107-141.

http://dx.doi.org/10.1177/1088868310367640

Kahneman, D. (2003). A perspective on judgment and choice mapping bounded rationality. American Psychologist, 58, 697-720.

http://dx.doi.org/10.1037/0003-066X.58.9.697

Kruglanski, A. W., \& Gigerenzer, G. (2011). Intuitive and deliberate judgments are based on common principles. Psychological Review, 118, 97. http://dx.doi.org/10.1037/a0020762

Kahneman, D. (2011). Thinking, fast and slow. New York: Farrar, Straus and Giroux.

Kahneman, D., \& Frederick, S. (2002). Representativeness revisited: Attribute substitution in intuitive judgement. In T. Gilovich, D. Griffin, \& D Kahneman (Eds.), Heuristics and biases: The psychology of intuitive judgment (pp. 49-81). Cambridge, UK: Cambridge University Press.

Martens, U., Ansorge, U., \& Kiefer, M. (2011). Controlling the unconscious attentional task sets modulate subliminal semantic and visuomotor processes differentially. Psychological Science, 22, 282 291. http://dx.doi.org/10.1177/0956797610397056

Mossbridge, J., Tressoldi, P., \& Utts, J. (2012). Predictive physiological anticipation preceding seemingly unpredictable stimuli: A meta-analysis. Frontiers in Psychology, 3, 390. http://dx.doi.org/10.3389/fpsyg.2012.00390

Pihlström, S. (2008). Panpsychism-A neglected jamesian alternative? Journal of Philosophical Research, 32, 319-347. http://dx.doi.org/10.5840/jpr20073238

Sherwood, S. J., \& Roe, C. A. (2003). A review of dream ESP studies conducted since the maimonides dream ESP program. Journal of Consciousness Studies, 10, 85-109.

Sio, U. N., \& Ormerod, T. C. (2009). Does incubation enhance problem solving? A meta-analytic review. Psychological Bulletin, 135, 94120. http://dx.doi.org/10.1037/a0014212

Stanovich, K. E. (1999). Who is rational? Studies of individual differences in reasoning. Mahwah, NJ: Elrbaum.

Staune, J. (2013). Towards non-physical realism. In A. Suarez, \& P. Adams (Eds.), Is science compatible with free will? (pp. 209-224). New York: Springer. http://dx.doi.org/10.1007/978-1-4614-5212-6 14

Storm, L., Tressoldi, P. E., \& Di Risio, L. (2010). Meta-analyses of free-response studies 1992-2008, assessing the noise reduction model in parapsychology. Psychological Bulletin, 136, 491-494. http://dx.doi.org/10.1037/a0019840

Storm, L., Tressoldi, P. E., \& Di Risio, L. (2012). Meta-analysis of ESP studies, 1987-2010: Assessing the success of the forced-choice design in parapsychology. Journal of Parapsychology, 72, 243-273.

Strick, M., Dijksterhuis, A., Bos, M. W., Sjoerdsma, A., van Baaren, R. B., \& Nordgren, L. F. (2011). A meta-analysis on unconscious thought effects. Social Cognition, 29, 738-762. http://dx.doi.org/10.1521/soco.2011.29.6.738

Strick, M., van Noorden, T. H., Ritskes, R. R., de Ruiter, J. R., \& Dijksterhuis, A. (2012). Zen meditation and access to information in the unconscious. Consciousness and Cognition, 21, 1476-1481. http://dx.doi.org/10.1016/j.concog.2012.02.010

Tamietto, M. et al. (2009). Unseen facial and bodily expressions trigger fast emotional reactions. PNAS, 106, 17661-17666. http://dx.doi.org/10.1073/pnas.0908994106

Tressoldi, P. E. (2011). Extraordinary claims require extraordinary evidence: The case of non-local perception, a classical and Bayesian review of evidences. Frontiers in Psychology, 2, 117. http://dx.doi.org/10.3389/fpsyg.2011.00117

Tressoldi, P., \& Khrennikov, A. (2012). Remote state preparation of mental information: A theoretical model and a summary of experimental evidence. Neuro Quantology, 10, 394-402.

Tressoldi, P. E., Rabeyron, T., Duggan, M., \& Bem, D. (submitted). Feeling the future: A meta-analysis of experiments on the anomalous anticipation of random future events.

Usher, M., Russo, Z., Weyers, M., Brauner, R., \& Zakay, D. (2011). The impact of the mode of thought in complex decisions: Intuitive decisions are better. Frontiers in Psychology, 2, 37. http://dx.doi.org/10.3389/fpsyg.2011.00037

Van den Bussche, E., den Noortgate, W., \& Reynvoet, B. (2009). Mechanisms of masked priming: A meta-analysis. Psychological Bulletin, 135, 452-477. http://dx.doi.org/10.1037/a0015329

Velmans, M. (2012). Introduction to monist alternatives to physicalism. Journal of Consciousness Studies, 19, 9-10. 


\section{Appendix}

\section{Synthetic Description of a Typical Experimental Protocol for the Study of S1_Nip Perception in a Ganzfeld Environment}

In a typical ganzfeld experiment, the "receiver" is left in a room relaxing in a comfortable chair with halved ping-pong balls over the eyes, and with a red light shining on them. The receiver is asked to keep his/her eyes open, and to wear headphones through which white or pink noise is played. The receiver is exposed to this state of mild sensory homogenization for about a half hour.

During this time a target usually a photograph or a short videoclip randomly drawn from a set of four possible targets (each as different from one another as possible) is projected on a screen located in a distant room isolated from the "receiver's" room without any possibility to receive sensory information (a common variant is the use of a "sender" who observes the chosen target).

During the ganzfeld stimulation period, the receiver verbally describes any impressions that come to mind. These "mentations" are recorded by the experimenter (who is also blind to the target) via an audio recording or by taking notes, or both. After the ganzfeld period ends, the receiver is taken out of the ganzfeld state and is presented with four photos or video clips, one of which was the target along with three decoys. The receiver is asked to choose which target best resembles the image sent by the distant sender.

The evaluation of a trial is based on (a) selection of one image by the receiver, based on his/her assessment of the similarity between his/her subjective impressions and the various target possibilities, possibly enhanced by listening to his/her mentation recorded during the session, or (b) an independent judge's assessment of similarity between the various targets and the participant's mentation recorded during the session. The results are then collected in the form of "hit rates" over many trials, (i.e., the proportion of trials in which the target was correctly identified). Because four possible targets are typically used in these studies, the chance hit rate is normally $25 \%$. After many repeated trials, hit rates that significantly exceed chance expectation are taken as evidence for nonlocal information transfer. Most of these experiments are now fully automated, eliminating the possibility of data recording errors.

\section{Synthetic Description of a Typical Experimental Protocol for the Study of S1_Nip Perception Recording Neuro and/or Psychophysiological Correlates}

Two paradigms are used: 1) randomly ordered presentations of arousing vs. neutral stimuli, or 2) guessing tasks for which the stimulus is the feedback about the participant's guess (correct vs. incorrect). In arousing vs. neutral stimulus paradigms, participants are shown, for example, a randomly intermixed series of violent and emotionally neutral photographs on each trial, and there is no a priori way to predict which type of stimulus will be viewed in the upcoming trial. In guessing tasks, on each trial participants are asked to predict randomly selected future stimuli (such as which of four cards will appear on the screen) and once they have made their prediction, they then view the target stimulus, which becomes feedback for the participant.
Because participants perform at chance on these tasks, guessing tasks generally create a random distribution of events producing separable physiological responses that reflect brief states of positive arousal (following feedback indicating a correct guess) and negative and/or lower arousal (following feedback indicating an incorrect guess). Regardless of the paradigm, physiological measures [skin conductance, heart rate, blood volume, respiration, electroencephalographic (EEG) activity, pupil dilation, blink rate, and/or blood oxygenation level dependent (BOLD) responses] are recorded throughout the session, and stimulus times are usually marked in the physiological trace itself. These continuous data are later portioned according to a pre-determined "anticipatory period" designated for analysis (generally 0.5 - $10 \mathrm{~s}$ preceding stimulus presentation, depending on the temporal sensitivity of the physiological measure and the intertrial interval). The portioned data are marked according to the type of stimuli they precede (arousing or neutral stimuli for the arousing vs. neutral paradigm, feedback indicating correct or incorrect guesses for the guessing paradigm). Pre-stimulus data are then compared across stimulus types.

It has been known for some time that arousing and neutral stimuli produce somewhat different post-stimulus physiological responses in humans. However, what is remarkable is that many of the studies examined here make the claim that, for instance, the same physiological measure that yields a differential post-stimulus response to two stimulus classes also yields a differential pre-stimulus response to those same stimulus classes, prior even to the random selection of the stimulus type by the computer. Authors of these studies often refer to the effect as presentiment (sensing an event before it occurs) or unexplained anticipatory activity; we favor the latter terminalogy as it describes the phenomenon without implying that the effect truly reflects a reversal of the usual forward causality. 